\title{
Desarrollo de las estructuras cognitivas del alumnado sobre el aire mediante actividades de investigación
}

\author{
Mónica Baptista \\ Instituto de Educação da Universidade de Lisboa.Lisboa.Portugal.mbaptista@ie.ulisboa.pt \\ ORCID: https:// orcid.org/0000-0003-1609-5764
}

Iva Martins

Instituto de Educação da Universidade de Lisboa. Lisboa. Portugal. ivamartins@ie.ulisboa.pt ORCID: https:// orcid.org/0000-0002-1485-2760

Teresa Conceição

Instituto de Educação da Universidade de Lisboa. Lisboa.Portugal.mariaconceicao@campus.ul.pt ORCID: https:// orcid.org/0000-0002-5893-5509

\section{Carolina Pipitone}

Departament d'Educació Lingüistica i Literària $i$ de Didàctica de les Ciències Experimentals i de la Matemàtica. Facultat de Formació del Professora. Universitat de Barcelona. Barcelona. España. cpipetone@ub.edu

ORCID: https:/ / orcid.org/0000-0002-4008-8727

[Recibido: 27 Julio 2019. Revisado: 20 Octubre 2019. Aceptado: 17 Enero 2020]

\begin{abstract}
Resumen: Este estudio pretende examinar el desarrollo de las estructuras cognitivas del alumnado de $4^{\circ}$ año, como resultado del uso de actividades de investigación, en la secuencia de clases que abordaban el concepto de aire. En el estudio han participado un total de 71 estudiantes en edades entre los 8 y 10 años. Los datos fueron recolectados a través de una prueba de asociación de palabras (Word Association Test - WAT) presentada al alumnado en dos momentos diferentes, antes y después de la secuencia de clases. Los resultados muestran modificaciones en las estructuras cognitivas de los estudiantes. En concreto se ha comprobado que, después de la secuencia de clases, las asociaciones más pobres entre las palabras-estímulo identificadas en el pre-test mostraron asociaciones con mayor superioridad en el post-test. Se destaca el análisis realizado a partir de las oraciones escritas por los estudiantes, en donde se observa que la naturaleza de estas asociaciones es más significativa para el tema de estudio, lo que revela una reorganización y desarrollo de las estructuras cognitivas del alumnado como resultado del uso de actividades de investigación en la secuencia de aula.
\end{abstract}

Palabras clave: Actividades de investigación; Estructuras cognitivas; Word Association Test, Aire.

\section{Inquiry in the development of students' cognitive structures}

\begin{abstract}
This study aims to evaluate the development of the cognitive structures of 4th grade students as a result of the use of inquiry activities in the sequence of lessons about the concept of air. The study was performed with a total of 71 students, aged from eight to ten years old. The data was collected through a Word Association Test (WAT) presented to students at two different moments, before and after the sequence of classes. The results reveal that the students' cognitive structures changed as a result of instruction. Specifically, it has been shown that, as a result of class sequence, the weakest associations between the stimulus words in the pretest appear in the posttest in a higher level of strength. The analysis of the sentences written by the students, that allows the disclosure of the nature of the associations, shows that they were more appropriated regarding the theme. This is indicative of a reorganization and development of the students' cognitive structures, as a result of the use of inquiry activities about the de concept of air.
\end{abstract}

Keywords: Inquiry; Cognitive structures; Word Association Test; Air. 
Para citar este artículo: Baptista, M., Martins, I., Conceição, T., y Pipitone, C (2020) Desarrollo de las estructuras cognitivas del alumnado sobre el aire mediante actividades de investigación. Revista Eureka sobre Enseñanza y Divulgación de las Ciencias 17(2), 2301. doi: 10.25267/Rev_Eureka_ensen_divulg_cienc.2020.v17.i2.2301

\section{Introducción}

El aire es un concepto difícil para los estudiantes porque, como señalan varios autores, el aire no es visible, no tiene sabor ni olor y se percibe principalmente a través de experimentos cinestésicos, esencialmente relacionados con su movimiento y con la transferencia de energía en forma de calor (Séré 1986). Así, a pesar de las experiencias cotidianas, los estudiantes no saben qué es el aire y son evidentes las dificultades que tienen para formar un modelo mental científico sobre este tema. Por ejemplo, en el estudio de Séré (1986), con 600 estudiantes de 11 años, los participantes describen el aire como el movimiento del viento y las corrientes de aire. Según Driver, Aosko, Leach y Scott (1994), para niños de hasta 6 años, el aire solo existe en forma de viento y varios alumnos en estas edades tienen dificultades para conceptualizar el aire como algo material. Además, muchos estudiantes describen el aire como «espacio vacío» (Krnel, Watson y Glažar 2005) y sin masa (Stavy 1988). Con respecto a la composición del aire, los estudiantes tienen dificultad para considerar el aire como una mezcla de gases y, según lo descrito por Johnson (1998), «aire» y «oxígeno» se usan como sinónimos. De manera similar, los estudiantes también tienen dificultades para comprender el concepto de «gas», que a menudo se asocia con combustibles fósiles (gas de encendedores, estufas, etc.) o como algo que es perjudicial para la salud (Séré 1985).

En este sentido, considerando la importancia de que los alumnos comprendan el concepto de aire, en los primeros años de escolaridad, es esencial recurrir a estrategias de enseñanza que les ayuden a aprenderlo. En particular, la investigación, ya que dadas sus características, puede promover un cambio en las percepciones de los estudiantes y, por lo tanto, constituir un medio importante para el desarrollo de sus estructuras cognitivas. Carlson, Humphrey y Reinhardt (2003) describen la actividad de investigación como una estrategia que involucra activamente al alumnado a elegir el camino a seguir y así, encontrar una o más soluciones con el objetivo de promover la relación entre conceptos y con ello la comprensión de los fenómenos. La actividad de investigación permite el desarrollo de actividades que exploran un problema o una situación del mundo actual, en donde la persona que investiga tiene un papel importante en la formulación de las preguntas, en la definición de sus objetivos y en la búsqueda de su propio camino hacia la resolución. Según Tweed (2009), un aspecto central de la actividad de investigación es precisamente crear oportunidades para que los estudiantes razonen, discutan, defiendan sus puntos de vista y construyan significados a partir de la relación entre los conceptos aprendidos.

Aunque los estudios indican que la actividad de investigación favorece el aprendizaje conceptual de los estudiantes en ciencias, todavía hay pocos que buscan saber cómo el uso de la actividad de investigación ayuda al alumnado a desarrollar sus estructuras cognitivas.

\section{Problema de investigación}

Este estudio tiene como objetivo abordar el siguiente problema de investigación: ¿Cuál es el efecto del uso de actividades de investigación en una secuencia de clases, sobre el concepto de aire, en el cambio de las estructuras cognitivas de los estudiantes de $4^{\circ}$ año? 


\section{Estructuras cognitivas del alumnado}

La importancia del conocimiento de las estructuras cognitivas (EC) del alumnado se relaciona con la forma en que realizan un aprendizaje significativo, basado en la construcción de nuevos conocimientos a partir de los conocimientos que poseen (Ausubel 1968). De esta manera, y como afirma Atabek-Yigit (2015), conocer las EC de los estudiantes ayuda a comprender y desarrollar el proceso de aprendizaje. Según esta autora, a través del conocimiento de las EC del alumnado, el profesorado puede comprender las concepciones que tienen los estudiantes, las relaciones que establecen entre los conceptos, las concepciones alternativas que poseen y, de esta manera, ayudarlos a lograr un aprendizaje significativo. Si bien existen otras definiciones de EC, en este estudio elegimos usar este término cuando nos referimos a las relaciones que se establecen entre conceptos, términos y/o procesos (Taber 2008), que se adquieren en las aulas y se almacenan en la memoria a largo plazo de forma jerárquica (Tsai 2001).

Tsai y Huang (2002) sostienen que la representación de EC se ha realizado utilizando diferentes métodos entre los cuales se incluyen las pruebas de asociación de palabras (en inglés Word Association Tests, WAT). Los WAT desarrollados inicialmente por Johnson (1967, 1969), han sido utilizados por varios investigadores en las últimas décadas para revelar las asociaciones que los estudiantes establecen entre conceptos y verificar si estas asociaciones son adecuadas y significativas (e.g. Bahar, Johnstone y Sutcliffe 1999, Baptista, Martins, Conceição y Reis 2019).

La implementación de un WAT consiste en la selección, por parte del docente o investigador, de conceptos relevantes (palabras-estímulo) relacionados con un tema científico, y en la escritura, por parte del alumnado, de palabras asociadas con estos mismos conceptos (palabras-respuesta), en un período de tiempo definido que puede variar (Bahar et al. 1999, Hovardas y Korfiatis 2006, Nakiboğlu 2008). De esta manera, un WAT informa sobre la cantidad y variedad de las respuestas del alumnado relacionadas con los conceptos clave, como así también sobre la complejidad de las asociaciones entre los conceptos, y su comprensión del tema. Sin embargo, los WAT tienen limitaciones porque no les permiten a los estudiantes conocer la naturaleza de las relaciones que estos establecen entre los términos, es decir, no les posibilita determinar cómo es que se establecen los vínculos entre las palabras-estímulo y las palabras-respuesta (Gunstone 1980). De esta forma, el análisis de las EC del alumnado debe complementarse con otros métodos, como entrevistas, escritura libre o construcción de mapas conceptuales (Nakiboğlu 2008, Derman y Eilks 2016).

Según Şendur y Toprak (2017), los estudios que usan WAT y los relacionados con la enseñanza de la ciencia se pueden dividir en dos grupos: aquellos que se aplican en un solo momento y que identifican las EC del alumnado (Derman y Eilks 2016), y aquellos que son aplicados en dos momentos diferentes, uno antes de la instrucción (pre-test) y otro después de la instrucción (post-test), los cuales evidencian cómo cambian las EC del alumnado en una secuencia de clases (e.g., Baptista et al. 2019, Şendur y Toprak 2017).

Por ejemplo, en el estudio desarrollado por Yildirir y Demirkol (2018), en el cual participaron 153 estudiantes de $6^{\circ}$ grado usaron un WAT sobre transformaciones físicas y químicas, utilizando seis palabras-estímulo. Los resultados mostraron que el alumnado tiene dificultades para distinguir los conceptos de transformación química y transformación física, además de identificar concepciones alternativas. Los estudiantes hacen pocas asociaciones entre los conceptos «partícula»y «transformación química», lo que indica que no entienden la transformación química como un fenómeno que ocurre a nivel sub-microscópico. De esta manera, y para favorecer un aprendizaje significativo en el alumnado, los autores sugieren que 
las prácticas pedagógicas incluyan un componente práctico y experimental que evidencie el nivel sub-microscópico de las transformaciones químicas y físicas.

\section{Actividad de investigación}

La actividad de investigación surge como una estrategia de enseñanza y aprendizaje con un gran potencial, ya que da lugar a un aprendizaje más profundo de ciencia y sobre ciencia (Lederman 2006, NRC 2000). Según la NRC (2000), la actividad de investigación involucra tareas multifacéticas que permiten: observaciones; identificación del problema; formulación de preguntas; investigación en diversas fuentes de información; la planificación de las investigaciones; la revisión de lo que ya se sabe sobre la experiencia; el uso de herramientas para analizar e interpretar datos; exploración, predicción y respuesta al problema; y comunicación de resultados.

Existen varios modelos para realizar la actividad de investigación en el aula. Uno de estos modelos son las 5E's (Bybee et al. 2006). Este modelo propone un ciclo de aprendizaje que consta de cinco fases: Engage, Explore, Explain, Elaborate and Evaluate. La fase de Engage (Motivación) tiene como objetivo motivar al alumnado a estudiar un tema en particular, buscando captar su interés y curiosidad. Para ello, el docente presenta una situación problemática, recurriendo a preguntas, textos o imágenes. Además, también es importante estimular el pensamiento del alumnado, permitiendo que el profesorado tenga una idea de lo que los estudiantes ya saben e identifique sus concepciones alternativas. Durante la fase de Explore (Exploración), los estudiantes cuestionan, hacen predicciones, formulan hipótesis, planifican una forma de evaluarlas, evalúan, registran observaciones y discuten los resultados con sus pares, comparan varias alternativas posibles y organizan la información recopilada. En la fase de Explain (Explicación), los estudiantes discuten los resultados, articulando sus observaciones con ideas teóricas, preguntas e hipótesis. Por lo tanto, es esencial que el docente promueva en el alumnado las explicaciones con sus propias palabras, que hagan explícitos los conceptos que surgieron de la experiencia, como así también, a utilizar los resultados para fundamentar sus explicaciones y escuchar críticamente las explicaciones de sus pares y del docente. En la fase de Elaborate (Elaboración), se promueve en el alumnado establecer relaciones con otros conceptos para luego, aplicar en nuevas situaciones los conceptos y capacidades utilizando ya sus definiciones formales. Finalmente, en la fase de Evaluate (Evaluación), los estudiantes evalúan sus propios conocimientos y la forma en que llegaron a ese conocimiento, así como las dificultades que tuvieron, lo que encontraron más interesante y cómo trabajaron en grupo.

\section{Metodología}

\section{Contexto de estudio}

Participaron en este estudio tres grupos de $4^{\circ}$ grado de primaria, con un total de 71 estudiantes (54\% de niñas y 46\% de niños), con edades entre 8 y 10 años. En Portugal, en las pautas curriculares para el $1^{\circ}$ ciclo, en concreto $4^{\circ}$ grado, se espera que el alumnado realice experimentos con aire para reconocer que el aire existe, que está en estado gaseoso y está compuesto de una mezcla de gases, y que incluye oxígeno (Ministério da Educação, 2004). Este estudio se centra en los efectos de una secuencia de clases (duración total de 540 minutos) en las EC del alumnado participante sobre el concepto del aire. Las clases fueron impartidas por tres profesores en dos escuelas diferentes. La experiencia en el aula del profesorado varía entre 18 y 31 años de experiencia profesional. 
Durante la secuencia de clases, los estudiantes trabajaron en grupos de cuatro integrantes y desarrollaron tres actividades que siguen un enfoque de actividad de investigación, y que fueron construidas en base al modelo 5E's (Bybee et al. 2006). En las tres actividades los alumnos recibieron una guía para orientar las respuestas al problema de investigación. En la Tabla 1 se presenta un esquema de las varias fases que constituyen el modelo de las 5E's, relacionándolas con las preguntas orientadoras presentes en el guión de los estudiantes. Antes de realizar este estudio, las autoras del artículo discutieron con el profesorado las tres actividades y construyeron conjuntamente las guías docentes de exploración de las actividades (Anexo).

Tabla 1. Fases que constituyen al modelo 5E's en cada una de las actividades y su conexión con el guion del alumno (Bybee et al., 2006).

\begin{tabular}{|c|c|c|c|}
\hline Fase modelo & $\begin{array}{c}\text { Actividad } 1 \\
\text { ¿E1 aire existe? }\end{array}$ & $\begin{array}{c}\text { Actividad } 2 \\
¿ \mathrm{E} 1 \text { aire tiene masa? }\end{array}$ & $\begin{array}{l}\text { Actividad } 3 \\
\text { ¿Cómo se puede identificar } \\
\text { el oxígeno en el aire? }\end{array}$ \\
\hline Explore & $\begin{array}{c}\text { Después del texto, la guía del } \\
\text { estudiante tiene un conjunto } \\
\text { de preguntas que les orientan } \\
\text { y permiten explorar el } \\
\text { problema: (1) Proporcionen } \\
\text { una respuesta a la pregunta } \\
\text { planteada. Con la ayuda del } \\
\text { profesor, investiguen } \\
\text { (enciclopedias y / o Internet) } \\
\text { de manera tal que les permita } \\
\text { saber más sobre la existencia } \\
\text { del aire y su estado físico; (2) } \\
\text { Planifiquen una experiencia } \\
\text { (¿Qué harán y cómo? ¿Qué } \\
\text { necesitarán?); (3) Realicen la } \\
\text { planificación. }\end{array}$ & $\begin{array}{l}\text { Después de la imagen, la guía } \\
\text { del estudiante tiene un } \\
\text { conjunto de preguntas } \\
\text { orientadoras que les permiten } \\
\text { explorar el problema: (1) } \\
\text { planifiquen una experiencia } \\
\text { (¿Qué harán y cómo? ¿Qué } \\
\text { necesitarán?); (2) Realicen la } \\
\text { planificación. }\end{array}$ & $\begin{array}{l}\text { Después de los cómics, la } \\
\text { guía del estudiante tiene un } \\
\text { conjunto de preguntas } \\
\text { orientadoras que les permiten } \\
\text { explorar el problema: (1) } \\
\text { Planifiquen una experiencia } \\
\text { (¿Qué harán y cómo? ¿Qué } \\
\text { necesitarán?); (2) Realicen la } \\
\text { planificación; (3) Identifiquen } \\
\text { el oxígeno producido usando } \\
\text { la prueba de la llama. }\end{array}$ \\
\hline Explain & $\begin{array}{l}\text { En esta etapa del guion de los } \\
\text { estudiantes se les pide que: (4) } \\
\text { Registren observaciones; (5) } \\
\text { Saquen conclusiones } \\
\text { respondiendo la pregunta ¿El } \\
\text { aire existe? }\end{array}$ & $\begin{array}{l}\text { En esta etapa del guion de los } \\
\text { estudiantes se les pide que: ( } 3 \text { ) } \\
\text { Registren observaciones; (4) } \\
\text { Saquen conclusiones } \\
\text { respondiendo la pregunta ¿El } \\
\text { aire tiene masa? }\end{array}$ & $\begin{array}{l}\text { En esta etapa del guion de los } \\
\text { estudiantes se les pide que: (4) } \\
\text { Registren observaciones; (5) } \\
\text { Saquen conclusiones } \\
\text { respondiendo la pregunta: } \\
\text { ¿Cómo se puede identificar el } \\
\text { oxígeno en el aire? }\end{array}$ \\
\hline Elaborate & $\begin{array}{l}\text { En esta etapa del guion de los } \\
\text { alumnos, se les pide que: }(6) \\
\text { Recopilen y analicen textos } \\
\text { sobre la existencia del aire. }\end{array}$ & $\begin{array}{l}\text { En esta etapa, se les pide a } \\
\text { dos estudiantes que: ( } 5) \\
\text { Exploren una simulación en } \\
\text { la masa de aire. }\end{array}$ & $\begin{array}{l}\text { En esta etapa, se solicita la } \\
\text { guía de los alumnos para: }(6) \\
\text { Exploren un vídeo sobre los } \\
\text { gases que componen el aire. }\end{array}$ \\
\hline Evaluate & \multicolumn{3}{|c|}{$\begin{array}{c}\text { Al finalizar la actividad, los estudiantes reflexionan sobre el trabajo desarrollado y responden } \\
\text { algunas preguntas como: ¿Qué aprendiste? ¿Qué dificultades sentiste? ¿Qué es lo que más te } \\
\text { gustó? ¿Qué es lo que menos te gustó? }\end{array}$} \\
\hline
\end{tabular}




\section{Recogida de dados}

En este estudio utilizamos el test de asociación de palabras, WAT, como un instrumento de recopilación de datos. El objetivo del WAT fue describir el efecto de una secuencia de clases sobre el aire en el desarrollo de las EC de los estudiantes. Para esto, el WAT fue presentado a los estudiantes en dos momentos del estudio: momento 1, pre-test (M1), fue realizado por el grupo de estudiantes tres semanas antes de la secuencia de clases; el momento 2, post-test (M2), fue realizado tres semanas después de la secuencia de clases. Las autoras del artículo seleccionaron cinco palabras-estímulo: aire, oxígeno, dióxido de carbono, nitrógeno y estado gaseoso. La selección de estas cinco palabras se realizó teniendo en cuenta dos criterios: (1) la palabra «aire» es una palabra-estímulo ya que es el concepto central y es importante conocer las asociaciones que los estudiantes hacen con esta palabra; (2) los conceptos clave relacionados con el aire están presentes en el plan de estudios y hacen referencia a sus principales componentes (oxígeno, nitrógeno y dióxido de carbono) y su estado físico a temperatura ambiente (estado gaseoso). Para garantizar la validez de las palabras-estímulo seleccionadas, se les pidió a tres docentes experimentados de $1^{\circ}$ ciclo que dieran su perspectiva sobre ellas, refiriéndose a su grado de idoneidad para los estudiantes de $4^{\circ}$ grado. Los tres docentes confirmaron que las palabras-estímulo seleccionadas eran apropiadas. Cada estudiante recibió un cuaderno de 5 páginas, cada una con una palabra-estímulo escrita en el siguiente orden: aire, oxígeno, dióxido de carbono, nitrógeno y estado gaseoso (Bahar et al. 1999, Derman y Ebenezer 2018). Se les pidió al grupo de estudiantes participantes que escribieran para cada palabra-estímulo todos los términos o palabras que recordaban que podrían estar asociados. Además, para conocer la naturaleza de las asociaciones, se les pidió que escribieran una oración en la que incluyeran cada una de las palabras-estímulo y sus palabras-respuesta (Nakiboğlu 2008). En los dos momentos, M1 y M2, los estudiantes tuvieron aproximadamente 10 minutos para realizar esta tarea (Bahar et al. 1999, Derman y Eilks 2016).

\section{Análisis de dados}

El análisis de datos de los WAT se realizó sobre la base del método del mapa de frecuencia (Bahar et al. 1999, Nakiboğlu 2008) que se describirá más adelante. Para garantizar la fiabilidad, los datos WAT fueron analizados de forma independiente por la primera y segunda autora. Para la comparación de los análisis, se utilizó como criterio el recuento del número total de las diferentes palabras-respuesta. Siguiendo el método de Miles y Huberman (1994), el consenso entre las autoras fue superior al 90\% en M1 y M2 (Tabla 2).

Tabla 2. Fiabilidad de los datos para M1 y M2.

\begin{tabular}{|c|c|c|}
\hline Palabra-estímulo & M1 (\%) & M2 (\%) \\
\hline Aire & 92 & 95 \\
\hline Oxígeno & 94 & 93 \\
\hline Dióxido de carbono & 96 & 91 \\
\hline Nitrógeno & 96 & 97 \\
\hline Estado gaseoso & 94 & 91 \\
\hline
\end{tabular}

Para construir la tabla de frecuencias (Bahar et al. 1999, Nakiboğlu 2008), se contabilizó el número de palabras-respuesta asociadas con cada palabra-estímulo. La tabla de frecuencia (Tabla 3) se construyó colocando las palabras-estímulo en la primera fila, en la segunda línea se colocaron los momentos de pre-test (M1) y post-test (M2), y las palabras-respuesta se 
colocaron en la primera columna. En el conteo de palabras solo se validaron como palabrasrespuestas a las relacionadas con el aire (alrededor del 60\% del total de palabras en pre-test y $75 \%$ en el post-test). Esto significa que las palabras que no estaban relacionadas con el concepto de aire no se consideraron: por ejemplo, la palabra «mar» asociada con la palabraestímulo «aire» (en portugués estas dos palabras solo difieren de una letra y suenan de la misma manera por lo que puede ser una asociación fonética hecha por el estudiante), la palabra «sombra» asociada con la palabra «aire», la palabra «carrera» asociada con la palabraestímulo «nitrógeno», etc. A partir de esta tabla, se construyeron dos mapas de estructuras cognitivas del alumnado, en los momentos M1 y M2 (Figuras 1 y 2). La construcción de los mapas se realizó colocando las palabras-estímulo dentro de un recuadro, y las palabrasrespuesta sin recuadro, y uniendo con flechas desde las palabras-estímulo a las palabrasrespuesta, siendo esta la dirección de las asociaciones hechas por el alumnado, es decir, de una palabra-estímulo hacia una palabra-respuesta o, eventualmente, hacia otra palabra-estímulo. El grosor de las flechas está determinado por el valor de frecuencia de la palabra-respuesta a la palabra-estímulo y representa la fuerza de las asociaciones: cuanto más ancha es la flecha, mayor es la frecuencia y, en consecuencia, mayor es la asociación. De esta manera, es posible tener una idea de la fuerza y la dirección de las asociaciones y obtener una relación entre los conceptos y las EC de los estudiantes (Derman y Eilks 2016, Nakiboğlu 2008). Con el fin de distinguir la naturaleza de los vínculos que el alumnado establece entre las palabras, y para suprimir la limitación del WAT presentadas por Gunstone (1980) y Nakiboğlu (2008), se presentó un análisis cualitativo de las oraciones escritas por los estudiantes, presentando ejemplos de las respuestas del alumnado.

\section{Resultados}

Con el objetivo de conocer el efecto de una secuencia de clases en la actividad de investigación sobre el aire en el desarrollo de las EC de los estudiantes de $4^{\circ}$ grado de primaria, se construyó la tabla de frecuencias (Tabla 3) que indica el número de palabras-respuesta para cada palabraestímulo en cada uno de los momentos (M1 y M2).

Tabla 3. Tabla de frecuencias para el WAT.

\begin{tabular}{|c|c|c|c|c|c|c|c|c|c|c|}
\hline \multirow[b]{3}{*}{ Palabra-respuesta } & \multicolumn{10}{|c|}{ Palabra-estímulo } \\
\hline & \multicolumn{2}{|c|}{ Aire } & \multicolumn{2}{|c|}{ Oxígeno } & \multicolumn{2}{|c|}{$\begin{array}{l}\text { Dióxido de } \\
\text { Carbono }\end{array}$} & \multicolumn{2}{|c|}{ Nitrógeno } & \multicolumn{2}{|c|}{ Estado gaseoso } \\
\hline & M1 & M2 & M1 & M2 & M1 & M2 & M1 & M2 & M1 & M2 \\
\hline Aire & - & - & 43 & 51 & 6 & 21 & 6 & 17 & 12 & 24 \\
\hline Oxígeno & 46 & 56 & - & - & 6 & - & 2 & - & 6 & 15 \\
\hline Dióxido de carbono & 8 & 38 & 3 & 4 & - & - & - & 4 & 4 & 6 \\
\hline Nitrógeno & 6 & 21 & - & - & - & 5 & - & - & - & - \\
\hline Estado Gaseoso & 17 & 16 & - & 12 & - & 9 & - & 8 & - & 9 \\
\hline Gas & - & - & 4 & 27 & 7 & 18 & 8 & 14 & 28 & 23 \\
\hline Respiración & 38 & 18 & 32 & 22 & 4 & 12 & - & - & - & - \\
\hline Viento & 50 & 62 & 6 & 7 & - & - & - & - & 12 & 10 \\
\hline Combustión & - & - & - & 12 & - & - & - & - & - & - \\
\hline Enciende la llama & - & - & - & 19 & - & - & - & - & - & - \\
\hline Partículas & - & 16 & - & 17 & - & 11 & - & 8 & - & 8 \\
\hline Agua oxigenada & - & - & - & 8 & - & - & - & - & - & - \\
\hline
\end{tabular}


Tabla 3. Continuación

\begin{tabular}{|c|c|c|c|c|c|c|c|c|c|c|}
\hline \multirow[b]{3}{*}{ Palabra-respuesta } & \multicolumn{10}{|c|}{ Palabra-estímulo } \\
\hline & \multicolumn{2}{|c|}{ Aire } & \multicolumn{2}{|c|}{ Oxígeno } & \multicolumn{2}{|c|}{$\begin{array}{l}\text { Dióxido de } \\
\text { Carbono }\end{array}$} & \multicolumn{2}{|c|}{ Nitrógeno } & \multicolumn{2}{|c|}{ Estado gaseoso } \\
\hline & M1 & M2 & M1 & M2 & M1 & M2 & M1 & M2 & M1 & M2 \\
\hline Agua & 12 & - & - & - & - & - & - & - & - & - \\
\hline Caliente & 16 & - & - & - & - & - & - & - & 10 & - \\
\hline Frío & 16 & - & - & - & - & - & - & - & - & - \\
\hline Cielo & 16 & - & - & - & - & - & - & - & - & - \\
\hline Árboles & 16 & 14 & 36 & 22 & 9 & 17 & 8 & - & - & - \\
\hline Tormenta & 2 & - & - & - & - & - & - & - & - & - \\
\hline Temperatura & 8 & 8 & - & - & - & 2 & - & - & - & - \\
\hline Pulmón & - & - & 4 & - & - & - & - & - & - & - \\
\hline Espacio & 4 & - & - & - & - & - & - & - & 12 & - \\
\hline Globo & - & - & 5 & - & - & - & - & - & - & - \\
\hline Fotosíntesis & - & - & - & - & 8 & 10 & - & - & - & - \\
\hline Escape de coche & - & - & - & - & 4 & - & - & - & - & - \\
\hline Nube & - & - & - & - & 2 & - & - & - & 8 & - \\
\hline Bebida gasificada & - & - & - & - & - & - & - & - & 10 & - \\
\hline Vapor de agua & - & - & - & - & - & - & - & - & 10 & - \\
\hline Bombona de oxígeno & - & - & 23 & - & - & - & - & - & - & - \\
\hline Vida & - & - & 2 & - & - & - & - & - & - & - \\
\hline
\end{tabular}

Los datos presentados en la Tabla 3 se utilizaron para construir los mapas de las EC de los estudiantes para cada uno de los momentos: pre-test/M1 (Figura 1) y post-test/M2 (Figura 2). Los intervalos de frecuencia han sido definidos teniendo en cuenta lo que describen Nakiboglu (2008) y Derman e Eilks (2016). Así, comenzamos considerando el valor de frecuencia más alto (50 en M1 y 62 en M2), que corresponde a la asociación de la palabra «viento» con la palabra-estímulo «aire» para los que se establecieron los intervalos de frecuencia más altos, $50 \geq \mathrm{f} \geq 41$ para M1 y $70 \geq \mathrm{f} \geq 61$ para $\mathrm{M} 2$, es decir, rangos que incluyen los valores de frecuencia más altos. El menor valor de frecuencia corresponde para M1 al rango $10 \geq \mathrm{f} \geq 1$ porque a este nivel de frecuencia aparecen todas las palabras-estímulo, siendo estas últimas «dióxido de carbono» y «nitrógeno» que aparecen solo en frecuencias inferiores a 10. Para M2, el último intervalo de frecuencia fue $30 \geq \mathrm{f} \geq 21$ ya que en este rango de frecuencias aparecen las últimas palabras-estímulo: «estado gaseoso» y «nitrógeno». A continuación se presentarán los resultados según los momentos de la intervención en el aula.

\section{Estructuras cognitivas iniciales: Pre-test}

En el M1, la EC del alumnado (Figura 1) se caracteriza por cinco niveles de asociación, siendo el nivel con mayores frecuencias de respuesta $(50 \geq \mathrm{f} \geq 41)$ el nivel más alto, que corresponde a la asociación más fuerte en la EC de los alumnos. Así, en este nivel aparecen dos de las cinco palabras-estímulo, asociadas entre sí en ambas direcciones, y la palabra-estímulo «aire» se asoció con la palabra-respuesta «viento». En el siguiente nivel, que incluye palabras con una frecuencia entre 40 y 31, los estudiantes asocian las palabras-estímulo («aire» y «oxígeno») con 
una palabra-respuesta común («respiración») y la palabra-estímulo «oxígeno» también se asoció con una nueva palabra-respuesta «árboles». En el Nivel 3 (30 $\geq \mathrm{f} \geq 21)$, aparece una nueva palabra-respuesta asociada con la palabra-estímulo «oxígeno»: «bombonas de oxígeno». Además, también hay una nueva palabra-estímulo, «estado gaseoso», a la que los estudiantes asocian la palabra-respuesta «gas», sin ninguna conexión entre las palabras ya mencionadas y, por lo tanto, este nivel se caracteriza por la presencia de dos islas aisladas. Sin embargo, en el siguiente nivel $(20 \geq \mathrm{f} \geq 11)$, estas dos islas están interconectadas por las palabras-respuesta «espacio», «caliente» y «agua», a las que los estudiantes asocian tanto «aire» como «estado gaseoso». Las palabras-estímulo restantes, «nitrógeno» y «dióxido de carbono» aparecen solo en el nivel de asociación más bajo, que se caracteriza por las frecuencias de asociación más bajas $(10 \geq \mathrm{f} \geq 1)$ y a las que varias palabra-respuesta permiten la interconexión entre todas las palabras-estímulo. Por ejemplo, las palabras ya incluidas en la EC del alumnado en niveles superiores, como «árboles», "gas», «árboles» y «oxígeno», se han asociado con la palabra «nitrógeno». La palabra-estímulo «dióxido de carbono» se usa para describir no solo las palabras existentes (por ejemplo, «oxígeno», «gas», «árboles», «respiración» etc.) sino también a nuevas palabras-respuesta (por ejemplo, «fotosíntesis», «escape de coche»y «nube»). 


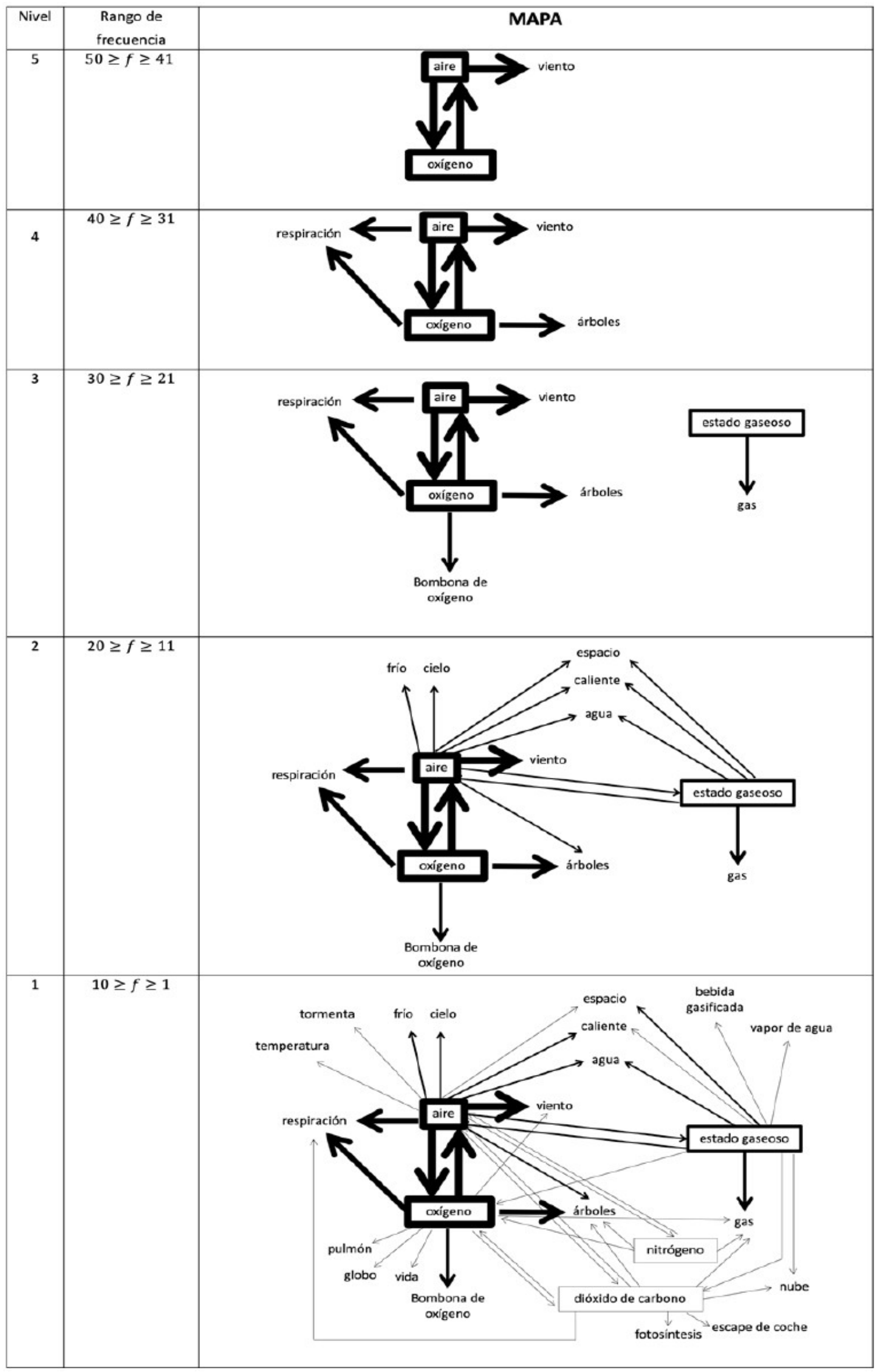

Figura 1. Mapa de las estructuras cognitivas del alumnado para el pre-test (M1). 
La naturaleza de las asociaciones se lleva a cabo mediante el análisis de las oraciones escritas por los estudiantes, algunas de las cuales se encuentran en la Tabla 4. La elección de las oraciones de la tabla se realizó a partir de la selección de aquellas que se consideraron representativas de las respuestas de los estudiantes. Por lo tanto, se seleccionaron las oraciones que ilustran las ideas del alumnado sobre las asociaciones entre palabras. Por cuestiones de organización y recopilación de resultados, solo se seleccionaron dos oraciones para cada asociación de palabras, que traducen las asociaciones más comunes, aunque hay otras oraciones con expresiones ligeramente diferentes. Por ejemplo, en la asociación aire-viento, las frases seleccionadas fueron «hoy hay viento y lo siento en mi cara» y «aire es el viento que sientes», pero los estudiantes escribieron otras frases similares, como «El viento es que el aire me golpea en la cara», «el viento es el propio aire», etc.

Tabla 4. Ejemplos de frases escritas por el alumnado en el pre-test (M1).

\begin{tabular}{|c|l|}
\hline Asociación & \multicolumn{1}{|c|}{ Ejemplo de frase } \\
\hline aire-viento & $\begin{array}{l}\text { El aire es el viento que sientes. } \\
\text { Hoy hay viento y lo siento en mi cara. }\end{array}$ \\
\hline aire-oxígeno & $\begin{array}{l}\text { El aire es oxígeno. } \\
\text { El oxígeno es el aire que respiras. }\end{array}$ \\
\hline $\begin{array}{c}\text { aire-respiración } \\
\text { oxígeno-respiración }\end{array}$ & $\begin{array}{l}\text { El oxígeno es el aire que respiras. } \\
\text { El oxígeno es lo que respiramos. }\end{array}$ \\
\hline oxígeno-árboles & $\begin{array}{l}\text { Los árboles dan el aire que es el oxígeno que respiramos. } \\
\text { Los árboles dan aire y oxígeno. }\end{array}$ \\
\hline oxígeno-bombonas de oxígeno & $\begin{array}{l}\text { Cuando entras en una ambulancia siempre hay una bombona de oxígeno. } \\
\text { Hoy fui a bucear hasta el fondo del mar con una botella en la espalda. }\end{array}$ \\
\hline estado gaseoso-gas & $\begin{array}{l}\text { El estado gaseoso es lo que tiene gas. } \\
\text { Mi estufa tiene una boquilla de gas que está en estado gaseoso. }\end{array}$ \\
\hline aire-caliente & $\begin{array}{l}\text { Cuando es invierno el aire esta frío. } \\
\text { En verano el aire es caliente. }\end{array}$ \\
\hline aire-frío & $\begin{array}{l}\text { El nitrógeno está en el aire. } \\
\text { El aire tiene oxígeno y nitrógeno. }\end{array}$ \\
\hline aire-dióxido de carbono & $\begin{array}{l}\text { El aire tiene dióxido de carbono. } \\
\text { El aire tiene dióxido de carbono y oxígeno. }\end{array}$ \\
\hline
\end{tabular}

\section{Estructuras cognitivas finales: Post-test}

En relación con el mapa de las EC de los estudiantes en el post-test (Figura 2), se caracteriza por la presencia de cinco niveles, pero con intervalos de frecuencia más altos, en comparación con el mapa en el pre-test (Figura 1). Por lo tanto, en el post-test, en el nivel con asociaciones más fuertes (Nivel 5, $70 \geq \mathrm{f} \geq 61$ ) aparece la palabra-estímulo «aire» asociada con la palabrarespuesta «viento». En el siguiente nivel $(60 \geq \mathrm{f} \geq 51)$ la palabra-estímulo «aire» ahora también se asocia con la palabra-estímulo: «oxígeno», que en el pre-test se encontraba en un nivel de asociación más débil $(50 \geq \mathrm{f} \geq 41)$. En el momento $\mathrm{M} 2$, en el nivel de frecuencia $50 \geq \mathrm{f} \geq 41$ aparece la conexión inversa, es decir, entre «oxígeno»y «aire», y en el Nivel 2 ( $40 \geq \mathrm{f} \geq 31$ ) la palabra-respuesta «aire» parece estar asociada con la palabra-estímulo «dióxido de carbono». 


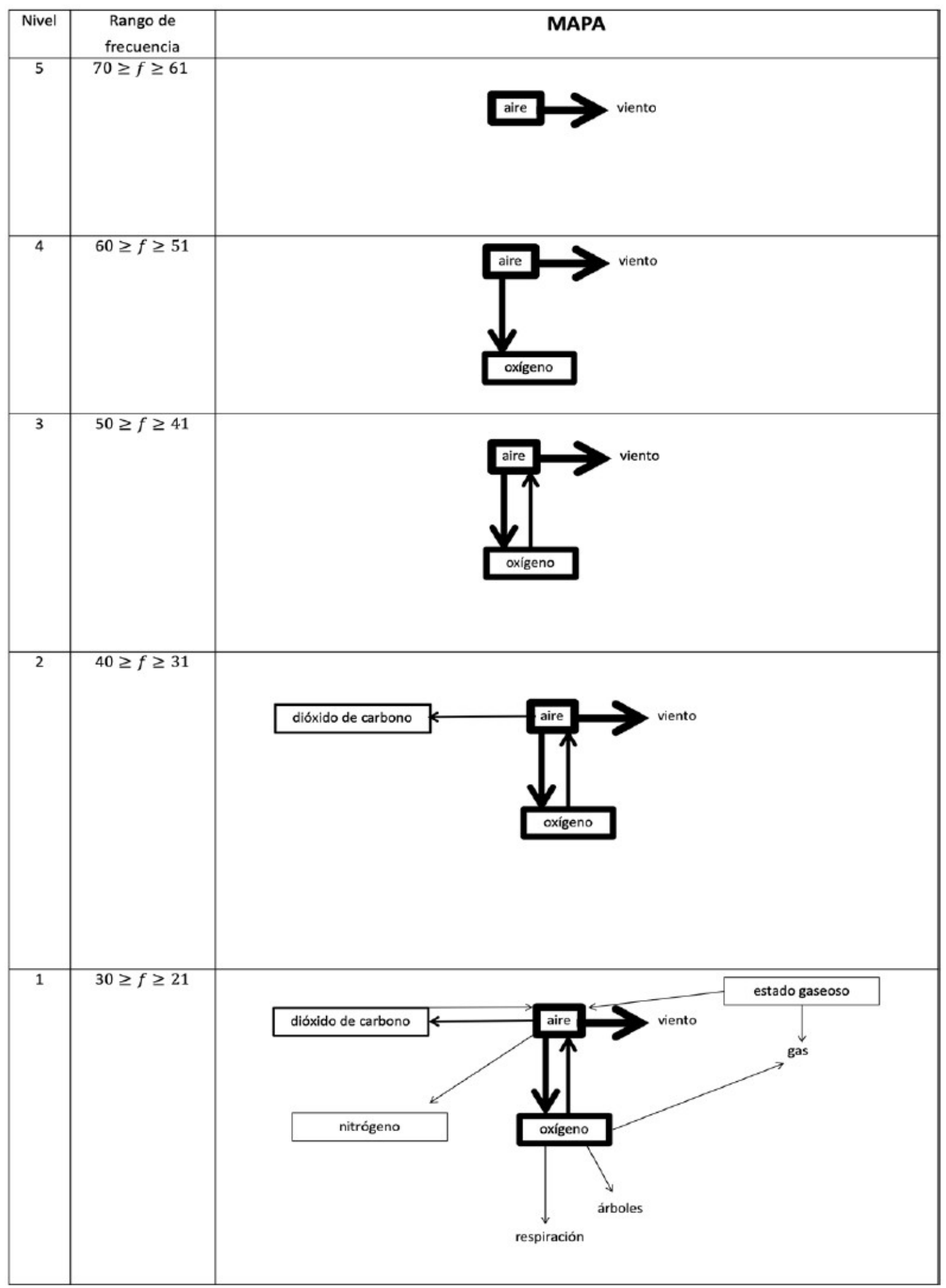

Figura 2. Mapa de las estructuras cognitivas del alumnado para el post-test (M2).

Finalmente, en el nivel más débil de asociación de la EC de los estudiantes en el post-test (30 $\geq \mathrm{f} \geq 21$ ), están presentes las palabras-estímulo restantes («nitrógeno» y «estado gaseoso»), así como nuevas palabras-respuesta («respiración», «gas» y «árboles»). Por lo tanto, se verifica en 
un primer análisis que, desde el pre-test al post-test, se evidencian cambios en la EC del grupo participante, lo que se refleja esencialmente en la fuerza de las asociaciones realizadas por los estudiantes: en el post-test aparecen las palabras-estímulo en niveles de asociación, en términos de frecuencias, más fuertes en comparación con el pre-test. Sin embargo, una vez más, es fundamental analizar la naturaleza de estos enlaces, para verificar si hubo cambios, y en la tabla 5 se presentan algunos ejemplos que representan las asociaciones más frecuentes que los alumnos hacen entre palabras-estímulo y palabras-respuesta.

Tabla 5. Ejemplos de frases escritas por el alumnado en el post-test (M2).

\begin{tabular}{|c|l|}
\hline \multicolumn{1}{|c|}{ Asociación } & \multicolumn{1}{|c|}{ Ejemplo de frase } \\
\hline aire-viento & $\begin{array}{l}\text { Veo el aire a través del viento. } \\
\text { En los días que hay mucho viento, el aire entra por debajo de la puerta de mi } \\
\text { casa. }\end{array}$ \\
\hline aire-oxígeno & $\begin{array}{l}\text { El aire tiene oxígeno y otros gases. } \\
\text { El aire no solo es oxígeno tiene otras cosas. }\end{array}$ \\
\hline aire-dióxido de carbono & $\begin{array}{l}\text { El oxígeno y el dióxido de carbono son parte del aire, pero hay más. } \\
\text { El aire tiene dióxido de carbono. }\end{array}$ \\
\hline $\begin{array}{c}\text { aire-nitrógeno } \\
\text { oxígeno-respiración } \\
\text { oxígeno-árboles }\end{array}$ & $\begin{array}{l}\text { El aire está compuesto de muchos gases, ejemplos de oxígeno, dióxido de } \\
\text { carbono y nitrógeno. } \\
\text { El nitrógeno es parte del aire. }\end{array}$ \\
\hline $\begin{array}{l}\text { Los árboles nos dan oxígeno por lo que respiramos y captan el dióxido de } \\
\text { carbono. } \\
\text { Los árboles y las flores son nuestros amigos porque nos dan oxígeno para } \\
\text { respirar. }\end{array}$ \\
\hline $\begin{array}{l}\text { aire-estado gaseoso } \\
\text { aire-gágeno-gas }\end{array}$ & $\begin{array}{l}\text { El aire está en estado gaseoso. } \\
\text { En el estado gaseoso tenemos aire que tiene peso. }\end{array}$ \\
\hline $\begin{array}{l}\text { En el estado gaseoso tenemos los gases. } \\
\text { Lleno el globo con aire y veo que el gas está atrapado allí. Puedo abrir el frasco } \\
\text { y ver si el aire tiene oxígeno porque enciende la llama. }\end{array}$ \\
\hline
\end{tabular}

\section{Análisis y discusión de resultados}

Los mapas de las EC del alumnado (Figuras 1 y 2) se caracterizan por tener cinco niveles de fuerza de asociación. Sin embargo, es notable que en el pre-test estos niveles son más débiles que en el post-test. Mientras que en el pre-test, el mapa de EC comienza con un rango de frecuencia de $50 \geq \mathrm{f} \geq 41$, en el post-test, el nivel de asociación más fuerte corresponde a los valores de las frecuencias $70 \geq \mathrm{f} \geq 61$. Con respecto al último nivel de frecuencia, definido por la aparición de todas las palabras-estímulo en el mapa cognitivo de los estudiantes, se verifica que en el pre-test esto corresponde a un nivel más bajo de asociación (10 $\geq \mathrm{f} \geq 1$ ), comparado con el post-test ( $30 \geq \mathrm{f} \geq 21$ ), lo que refleja una mayor fuerza de asociación entre los conceptos en el post-test (Nakiboğlu 2008).

También es evidente, en particular en el pre-test (Figura 1), la aparición de muchas palabras que resultan de sensaciones que los estudiantes experimentan en su vida cotidiana, como el viento, que es el resultado del «pensamiento natural» que es muy rudimentario para los niños, y carecen de interpretaciones más elaboradas (Guidoni 1985). De acuerdo con las frases escritas por el 96\% de los estudiantes que hicieron esta asociación, el aire está asociado con su movimiento y lo expresa a través de la palabra «viento», sin distinguir estos dos conceptos. En el post-test, aunque la asociación más fuerte es «aire» con «viento», la naturaleza de esta conexión es diferente (Tabla 5). Por ejemplo, y tal como describen 34 alumnos (55\% de los alumnos que hicieron esta asociación), comprenden que el aire y el viento no son lo mismo, 
pero que la manera de sentir el aire es a través del viento. Según lo descrito por Driver et al. (1994), los niños y niñas de hasta 6 años tienen dificultades para conceptualizar el aire como una sustancia material pudiendo realizar la conceptualización a partir de los 7 y 8 años, y según Séré (1986) los niños y niñas focalizan de manera espontánea en el movimiento del aire, pero tienen dificultad de conceptualizar el aire en un estado de equilibrio, sin movimiento, es decir, sin poder sentirlo. A partir de esta edad, y según Brook, Driver y Hind (1989, citado por Driver et al. 1994), los niños comienzan a entender el aire como materia que ocupa espacio, aunque generalmente no conceptualizan el hecho de, por ejemplo, tener masa.

Con respecto a la asociación que los estudiantes hacen entre las palabras-estímulo «aire y oxígeno», los mapas cognitivos muestran que esta asociación es ligeramente más fuerte en el post-test. Sin embargo, el análisis de la naturaleza de las asociaciones en los dos momentos es revelador de la alteración de sus concepciones iniciales. Por lo tanto, en el pre-test, el $83 \%$ del alumnado que hizo esta asociación no distingue «aire» de «oxígeno» y consideran, por ejemplo, que «aire es oxígeno» o que «oxígeno es el aire que se respira» (Tabla 4), tal como se describe en la literatura con respecto a las dificultades del alumnado para distinguir estos dos términos (Johnson 1998). Sin embargo, en el post-test, las frases de los alumnos revelan que la secuencia de las clases de las actividades de investigación tuvo un efecto positivo en la comprensión del tema, donde 30 estudiantes $54 \%$ de los estudiantes que hicieron esta asociación) explican que el aire es una mezcla de gases, incluido el oxígeno. Esta idea se ilustra en algunas frases escritas por los estudiantes, como «el aire no es solo oxígeno, hay otras cosas» y «el aire tiene oxígeno y otros gases» (Tabla 5). De esta manera, es evidente no solo la comprensión de los conceptos de «aire» y «oxígeno» sino también del aire como una mezcla de gases.

Otras asociaciones de palabras que aparecen tanto en el pre-test como en el post-test son, por ejemplo, entre las palabras «oxígeno», «respiración» y «árboles». Sin embargo, en el post-test, los estudiantes muestran que han adquirido conocimiento sobre el papel de los árboles en la absorción de dióxido de carbono y en la producción de oxígeno. Aunque no era uno de los objetivos, esta secuencia de clases permitió cambiar concepciones alternativas anteriores, consolidar el conocimiento y mejorar el lenguaje científico de los estudiantes. Por lo tanto, los estudiantes revelaron comprender que el papel de los árboles es fundamental para la respiración («los árboles nos dan oxígeno para que respiremos y captan el dióxido de carbono»), lo que difiere mucho de las concepciones iniciales del grupo de estudiantes («el oxígeno es el aire que respiras» y «los árboles lanzan el aire, que es el oxígeno que respiramos»).

Respecto a la palabra «estado gaseoso», aparece en la EC de los estudiantes, tanto en el pretest (Figura 1) como en el post-test (Figura 2), a un nivel de frecuencia correspondiente a $30 \geq$ $\mathrm{f} \geq 21$, pero el análisis de las frases escritas por el alumnado revela una vez más que las asociaciones hechas por el grupo de estudiantes tienen una naturaleza completamente diferente. En el pre-test, el 39\% de los estudiantes asociaron la palabra-estímulo «estado gaseoso» con la palabra-respuesta «gas» y algunas de las frases escritas por los estudiantes que hicieron esta asociación fueron: «el estado gaseoso es lo que tiene gas» y «la estufa tiene una boquilla de gas que está en estado gaseoso» (Tabla 4). Estos resultados están de acuerdo con lo que se describe en la literatura, no solo con respecto a la incapacidad del alumnado para entender el concepto de «gas», porque no es un término ampliamente utilizado, sino más bien a la asociación que hacen generalmente entre «gas» y combustibles fósiles (Séré 1985). Ya en el post-test (Tabla 5), 24 estudiantes hicieron la asociación entre el estado gaseoso y el aire, y de estos, el 75\% identificó que el aire estaba en estado gaseoso. Sin embargo, solo el 4\% de los estudiantes asociaron el estado físico con la existencia de masa, como por ejemplo la siguiente 
frase: «en el estado gaseoso tenemos el aire que tiene peso», lo que evidencia la comprensión de que la materia en estado gaseoso, en este caso el aire, tiene masa, considerando que utiliza el peso como sinónimo de masa ya que no distingue entre los dos conceptos.

Una de las diferencias más evidentes y significativas entre los mapas del pre-test y post-test es el nivel en el que aparecen todas las palabras-estímulo, es decir, los niveles que determinan dónde termina el mapa cognitivo. En el mapa cognitivo correspondiente al pre-test (Figura 1), esto solo ocurre en un nivel de asociación muy débil (con frecuencia de respuestas por debajo de 10) y este nivel se caracteriza por la aparición de las últimas palabras-estímulo: «nitrógeno» y «dióxido de carbono». Sin embargo, los pocos estudiantes (6-8 estudiantes) que son capaces de asociar estas palabras-estímulo lo hacen correctamente, como lo demuestran las frases que escribieron: «el aire tiene oxígeno y nitrógeno» y «el aire tiene dióxido de carbono y oxígeno», reconociendo que el aire se compone por más de una sustancia. En el post-test (Figura 2), estas mismas palabras-estímulo aparecen en un nivel, en términos de frecuencias, superior y se asocian de manera más objetiva con las palabras-estímulo ya presentes en sus EC, es decir, sin estar inmersas en una gran variedad de palabras-respuesta, como ocurre en el pre-test. Por lo tanto, en el post-test, la EC del alumnado se caracteriza por la aparición de todas las palabrasestímulo en el nivel correspondiente a $30 \geq \mathrm{f} \geq 21$, es decir, dos niveles por encima del pretest. Además, el análisis de la naturaleza de las asociaciones realizadas por los estudiantes revela que la instrucción permitió la reorganización de sus EC, la evolución de algunas concepciones alternativas y el establecimiento de relaciones en consonancia con la temática enfocada, como se ilustra a través de frases como «el aire se compone de muchos gases, los ejemplos son oxígeno, dióxido de carbono y nitrógeno» en el cual se relacionan cuatro de las cinco palabras-estímulo correctamente. Por lo tanto, y aunque no usan explícitamente la palabra mezcla, este concepto está implícito cuando afirman que el aire está compuesto de muchos gases (oxígeno, dióxido de carbono, nitrógeno) y está en estado gaseoso.

\section{Conclusiones}

Entender qué es el aire y qué este existe es difícil para los estudiantes de primaria (Séré 1986). Por lo tanto, es fundamental que el docente utilice estrategias de enseñanza, como la actividad de investigación (NRC 2000), que permite al alumnado comprender el concepto de aire y, por lo tanto, lograr un aprendizaje significativo. En este estudio, se utilizó una actividad de investigación, siguiendo el modelo de las 5E's (Bybee et al. 2006), para analizar el efecto de una secuencia de clases sobre el aire en el desarrollo de las estructuras cognitivas del alumnado de $4^{\circ}$ grado. El uso de un WAT (Bahar et al. 1999, Nakiboğlu 2008) aplicado en dos momentos diferentes, antes y después de la secuencia de clases, reveló modificaciones en las estructuras cognitivas de los estudiantes, es decir, las asociaciones que establecen entre conceptos y el análisis de las oraciones escritas por los estudiantes permitieron averiguar la naturaleza de tales asociaciones, en particular si estas son apropiadas y significativas. Los resultados muestran que la fuerza de asociación de los conceptos es menor en el pre-test, lo que se traduce en frecuencias de respuestas más bajas y que la estructura cognitiva subyacente sea muy rudimentaria, sin concepciones muy elaboradas o correctas desde el punto de vista científico. Esencialmente, la estructura cognitiva del alumnado en el pre-test se caracteriza por asociaciones con sus experiencias sensoriales cotidianas, y la naturaleza básica de estas asociaciones se verificó mediante el análisis de oraciones escritas. Muchas de las dificultades identificadas en el pre-test concuerdan con las descritas en la literatura: por ejemplo, la naturaleza del aire como sustancia material que existe en un estado de equilibrio; «aire»y «oxígeno» son palabras que se usan con el mismo significado (Johnson, 1998). Sin embargo el planteamiento de una secuencia de clases sobre el concepto de aire, utilizando la actividad de 
investigación, tuvo un efecto positivo en el desarrollo de la estructura cognitiva del alumnado. Así, en el post-test, se observaron asociaciones más fuertes entre las palabras-estímulo, que aparecen, en su totalidad, dos niveles por encima del pre-test. Además, se verificó mediante el análisis de las frases escritas por el alumnado que la naturaleza de estas asociaciones es más apropiada para el tema en estudio, lo que revela una reorganización y desarrollo de las estructuras cognitivas del alumnado como resultado de la instrucción con el uso las actividades de investigación. Por lo tanto, este trabajo nos permite concluir que este tipo de actividades relacionadas al concepto de aire tuvieron, en general, un efecto positivo en el desarrollo de las estructuras cognitivas de los estudiantes sobre este tema. Sin embargo, se constató que conceptos que implican explicaciones a nivel microscópico, por ejemplo el hecho de que el aire tenga masa, no han sido desarrollados por la mayoría de los estudiantes. De acuerdo con los resultados obtenidos, los estudiantes pueden asociar el aire con el estado gaseoso, pero tienen dificultades para entender que este estado físico de la materia tiene masa, lo que también describe Séré (1985). En este sentido, y dado que este era el objetivo de la Actividad 2 , esta dificultad puede estar relacionada con la naturaleza misma de la actividad. De las tres actividades realizadas, es probablemente la menos atractiva desde el punto de vista de los estudiantes en el componente de laboratorio. Además, como se describe en la literatura (Driver et al. 1994), el hecho de que los estudiantes tengan la percepción de que un globo lleno de aire, cuando se deja caer, tarda más tiempo en llegar al suelo que un globo vacío les dificulta conceptualizar que el aire tiene masa. Aunque comprobaron mediante mediciones en una báscula que efectivamente un globo lleno de aire tenía una masa mayor que un globo vacío, no fue posible observar un cambio en las concepciones iniciales de los estudiantes. De manera similar, después de realizar el componente de experimentación, es decir, en la fase de Elaborate (modelo de 5E's), los estudiantes exploraron una simulación que no les permitió obtener información diferente a la obtenida a través de la actividad de laboratorio, y puede haber condicionado el aprendizaje del concepto. en cuestión, por lo que será importante mejorarlo. También es posible concluir que el WAT, asociado al análisis de la naturaleza de las asociaciones realizadas por los estudiantes, es un método adecuado para investigar y evaluar las estructuras cognitivas y sus modificaciones como resultado de los procesos de enseñanzaaprendizaje.

\section{Referencias}

Atabek-Yigit E. (2015) Exploring the relationship between cognitive structure outcomes and test achievements of preservice science teachers on chemical bonding via flow mapping. Journal of Baltic Science Education 14(4), 524-534.

Ausubel D. P. (1968) Educational psychology. A cognitive view. New York: Holt, Rinehart and Winston, Inc.

Bahar M., Johnstone A. H., Sutcliffe R. G. (1999) Investigation of students' cognitive structure in elementary genetics through word association tests. Journal of Biological Education 33(3), 134-141. https://doi.org/10.1080/00219266.1999.9655653

Baptista M., Martins I., Conceição T., Reis P. (2019) Multiple representations in the development of the students' cognitive structures about the saponification reaction. Chemistry Education Research and Practice 20(4), 760-771. https://doi.org/10.1039/C9RP00018F

Brook A., Driver R., Hind D. (1989) Progression in science: The development of pupils' understanding of physical characteristics of air across the age range 5-16 years. United Kingdom: Center for Studies in Science and Mathematics Education, University of Leeds. 
Bybee R. W., Taylor J. A., Gardner A., Van Scotter P., Powell C., Westbrook A., Landes N. (2006) The BSCS 5E instructional model: Origins and effectiveness. Colorado Springs, CO: BSCS.

Carlson L., Humphrey G., Reinhardt K. (2003) Weaving science inquiry and continuous assessment. Thousand Oaks, CA: Corwin Press.

Derman A., Ebenezer J. (2018) The effect of multiple representations of Physical and Chemical changes on the development of primary pre-service teachers cognitive structures. Research in Science Education, 1-27. https://doi.org/10.1007/s11165-0189744-5

Derman A., Eilks I. (2016) Using a word association test for the assessment of high school students' cognitive structures on dissolution. Chemistry Education Research and Practice 17(4), 902-913. https://doi.org/10.1039/c6rp00084c

Driver R., Asoko H., Leach J., Mortimer E., Scott P. (1994) Constructing scientific knowledge in the classroom. Educational Researcher 23(7), 5-12. https://doi.org/10.4324/9780203464021

Guidoni P. (1985) On natural thinking. European Journal of Science Education 7(2), 133-140. https://doi.org/10.1080/0140528850070204

Gunstone R. F. (1980) Word association and the description of cognitive structure. Research in Science Education 10(1), 45-53. https://doi.org/10.1007/bf02356308

Hovardas T., Korfiatis K. J. (2006) Word associations as a tool for assessing conceptual change in science education. Learning and Instruction 16(5), 416-432. https://doi.org/10.1016/j.learninstruc.2006.09.003

Johnson P. (1998) Children's understanding of changes of state involving the gas state, Part 1: Boiling water and the particle theory. International Journal of Science Education 20(5), $567-$ 583. https://doi.org/10.1080/0950069980200505

Johnson P. E. (1967) Some Psychological Aspects of Subject-Matter Structure. Journal of Educational Psychology 58(2), 75-88.

Johnson P. E. (1969) On the Communication of Concepts in Science. Journal of Educational Psychology 60(1), 32-40.

Krnel D., Watson R., Glažar S. A. (2005) The development of the concept of 'matter': A cross-age study of how children describe materials. International Journal of Science Education 27(3), 367-383. https://doi.org/10.1080/09500690412331314441

Lederman L. G. (2006) Syntax of the nature of science within inquiry and science instruction. In L. B. Flick, N. G. Lederman (Eds.), Scientific inquiry and nature of science. Implications for teaching, learning, and teacher education. Dordrecht: Springer.

Miles M. B., Huberman A. M. (1994) Qualitative Data Analysis: An Expanded Sourcebook. Thousand Oaks, CA: Sage Publications.

Ministério da Educação (2004). Programa 1. ciclo do ensino básico. Lisboa: Ministério da Educação.

Nakiboğlu C. (2008) Using word associations for assessing non major science students' knowledge structure before and after general chemistry instruction: The case of atomic structure. Chemistry Education Research and Practice 9(4), 309-322. https://doi.org/10.1039/b818466f 
NRC (National Research Council) (2000) Inquiry and the National Science Education Standards. Washington, DC: National Academy Press.

Şendur G., Toprak M. (2017) An investigation of changes in the cognitive structures of 11th grade students using the word association test: the case of chemical equilibrium. Journal of Turkish Studies 12 (17), 411-436. https://doi.org/10.7827/turkishstudies.11911

Séré M. G. (1985) The Gasesous State. In R. Driver, E. Guesne, A. Tiberghien (Eds.), Childrens' Ideas in Science (pp. 105-123). Philadelphia: Open University Press.

Séré M. G. (1986) Children's conceptions of the gaseous state, prior to teaching. European Journal of Science Education 8(4), 413-425. https://doi.org/10.1080/0140528860080408

Stavy R. (1988) Children's conception of gas. International Journal of Science Education 10(5), 553560. https://doi.org/10.1080/0950069880100508

Taber K. S. (2008) Exploring conceptual integration in student thinking: evidence from a case study. International Journal of Science Education 30(14), 1915-1943.

Tsai C. C. (2001) Probing students' cognitive structures in science: the use of a flow map method coupled with a meta-listening technique. Studies in Educational Evaluation 27(3), 257-268. https://doi.org/10.1016/s0191-491x(01)00029-3

Tsai C.-C., Huang C.-M. (2002) Exploring students' cognitive structures in learning science: a review of relevant methods. Journal of Biological Education 36(4), 163-169. https://doi.org/https://doi.org/10.1080/00219266.2002.9655827

Tweed A. (2009) Designing Effective Science Instruction. What Works in Science Classrooms. Arlington, Virginia: NSTA Press.

Yildirir H. E., Demirkol H. (2018) Revealing students' cognitive structure about physical and chemical change: use of a word association test. European Journal of Education Studies 4(1), 134-154. https://doi.org/10.5281/zenodo.1156414 


\section{Anexo1: Guion de exploración de actividades para el profesorado}

Actividad 1

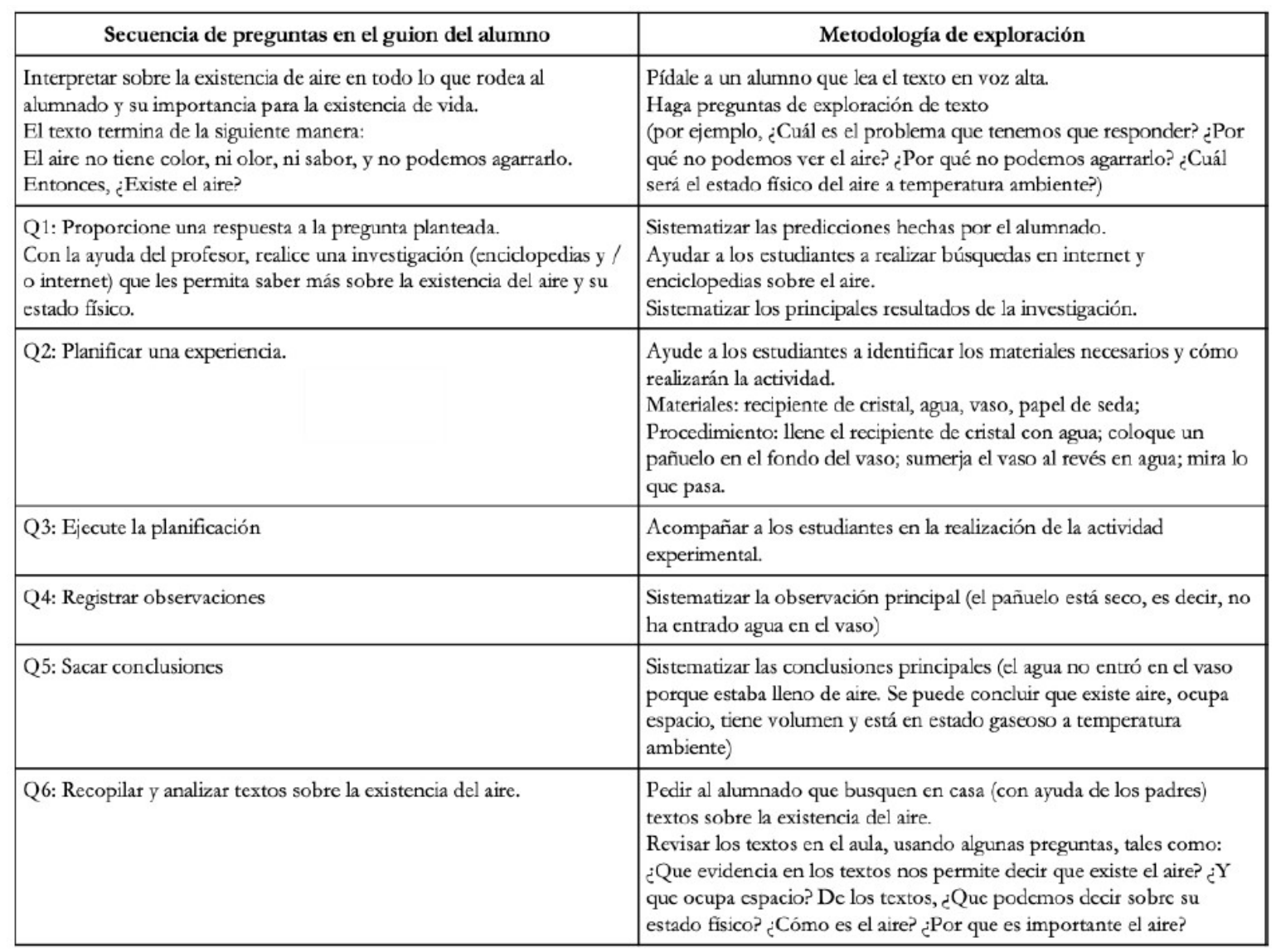




\section{Actividad 2}

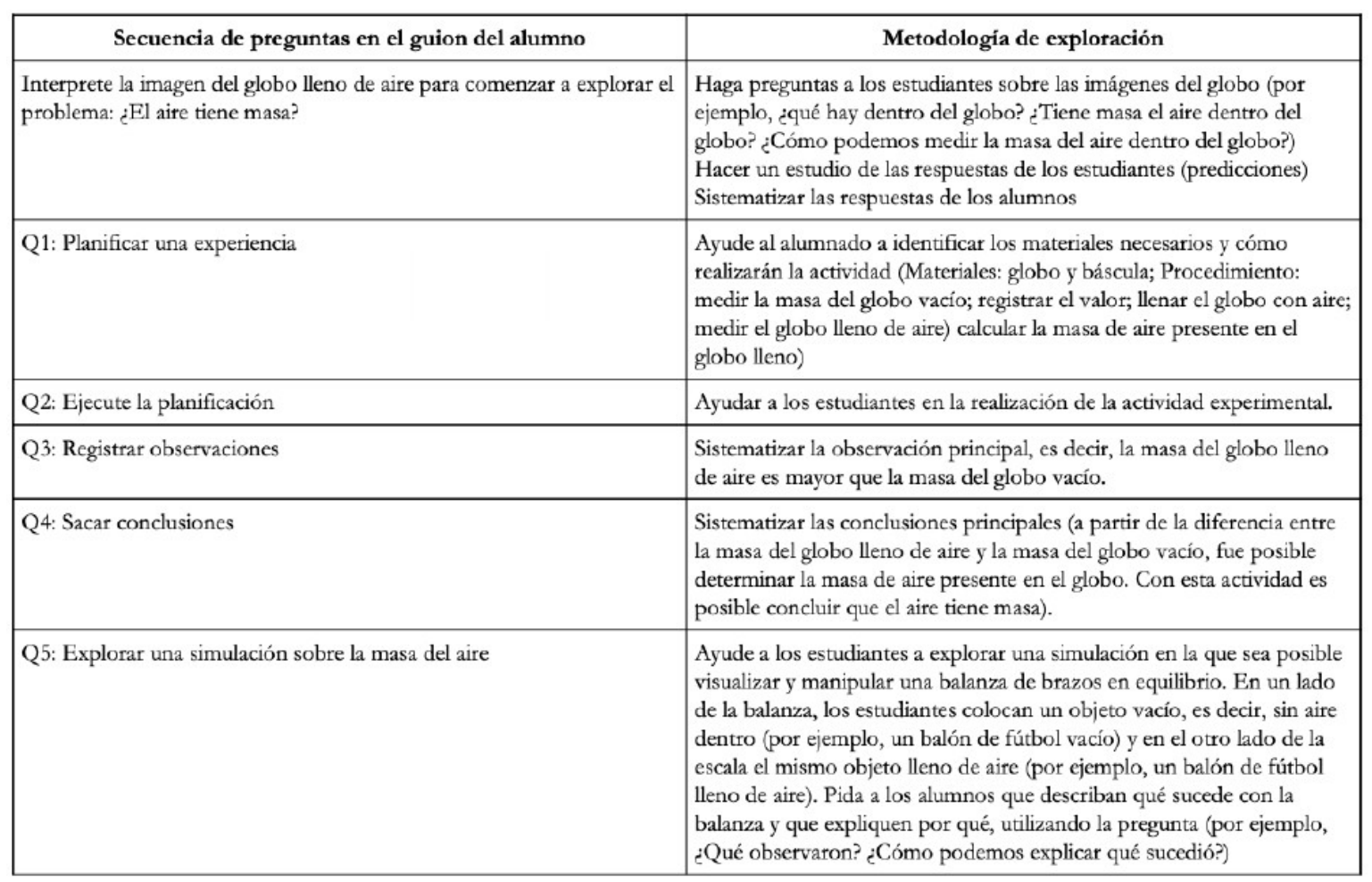




\section{Actividad 3}

\begin{tabular}{|c|c|}
\hline Secuencia de preguntas en el guion del alumno & Metodología de exploración \\
\hline Q1: Planificar una experiencia & $\begin{array}{l}\text { Ayude al alumnado a identificar los materiales necesarios y a cómo } \\
\text { realizarán la actividad (Materiales: peróxido de oxígeno ( } 9 \% \mathrm{~m} / \mathrm{m}) \text {, vaso } \\
\text { de precipitados de } 50 \mathrm{ml} \text {, yoduro de potasio, bolsa con cierre de } 1 \mathrm{~L} \text {; } \\
\text { Procedimiento: Mida con vaso de precipitados de } 50 \mathrm{ml} \text { de peróxido de } \\
\text { oxígeno, agregue el peróxido de oxígeno en la bolsa con cierre zip, } \\
\text { agregue una cuchara de café de yoduro de potasio, cierre la bolsa con } \\
\text { cierre zip y agite, observe lo que sucede, abra la bolsa y coloque una } \\
\text { cerilla dentro de la bolsa incandescente, observa lo que pasa) }\end{array}$ \\
\hline Q2: Ejecute la planificación & Ayudar al alumnado en la realización de la actividad experimental \\
\hline Q3: Registrar observaciones & $\begin{array}{l}\text { Sistematice las principales observaciones (en la bolsa se observa que se } \\
\text { forma un gas, el volumen de la bolsa aumenta, y que este gas ilumina la } \\
\text { llama de una cerilla incandescente) }\end{array}$ \\
\hline Q4: Sacar conclusiones & $\begin{array}{l}\text { Sistematice las principales conclusiones (peróxido de hidrógeno en la } \\
\text { bolsa descompuesto en agua y oxígeno. Al colocar una cerilla incesante } \\
\text { en la bolsa, la llama se encendió y se puede concluir que se formó } \\
\text { oxígeno. Extrapolando a la existencia de oxígeno en el aire, se puede } \\
\text { concluir que el oxígeno es uno de sus gases constituyentes porque se } \\
\text { observa la combustión de fósforo en el aire, es decir, el oxígeno } \\
\text { alimenta la combustión de fósforo }\end{array}$ \\
\hline
\end{tabular}

